\title{
NOUVELLE
}

\section{L'adaptation optimale du mouvement humain au milieu gravitaire}

Jérémie Gaveau, Bastien Berret, Charalambos Papaxanthis

Inserm U1093, laboratoire cognition, action et plasticité sensorimotrice ; université de Bourgogne-Franche Comté, Institut Marey, 64, rue de Sully, 21000 Dijon, France. jeremie.gaveau@u-bourgogne.fr

\section{La théorie computationnelle du contrôle moteur}

Chaque jour, notre système nerveux central engage de manière automatique des processus sophistiqués nous permettant d'exécuter des mouvements volontaires d'une grande complexité. Cette complexité, le plus souvent silencieuse, est durement révélée lorsque notre système nerveux n'est plus capable de réaliser un contrôle moteur parfait, par exemple à la suite d'un accident vasculaire cérébral. L'étude du mouvement humain permet la caractérisation de ces processus silencieux et inaccessibles.

Après deux décennies de recherche sur le mouvement, émerge la théorie computationnelle du contrôle moteur qui permet d'unifier une grande variété de résultats obtenus empiriquement [1]. Cette théorie utilise des approches mathématiques pour formaliser/clarifier les problèmes posés par le contrôle des mouvements du corps et ainsi proposer et tester les possibles solutions que notre système nerveux central pourrait avoir développées. Le concept le plus largement reconnu est, sans conteste, celui de modèle interne. D'un point de vue neurobiologique, un modèle interne consiste en un réseau d'activations neuronales permettant d'imiter (de modéliser) le fonctionnement d'un capteur sensoriel, d'un effecteur (un segment corporel) ou encore d'une loi physique à laquelle notre corps est soumis (comme la force gravitaire ou l'inertie). Il en découle donc une faculté de prédiction par notre système nerveux central, le rendant capable d'anticiper les effets sensoriels et physiques produits par l'envoie d'une commande motrice (par exemple des activations musculaires).

Le cas de l'adaptation du mouvement au milieu gravitaire

Grâce à de multiples études de neurophysiologie réalisées chez le macaque [2] et chez l'homme [3], un consensus sur l'existence d'un modèle interne de la gravité terrestre dans le cerveau des primates a été trouvé. D’après ce modèle, il est possible de prévoir l'effet permanent de la gravité terrestre sur les mondes animé et inanimé, par exemple la chute d'objets ou la perte d'équilibre. Cependant, une question reste débattue dans le domaine du contrôle moteur: comment cette représentation interne de la gravité est-elle utilisée pour contrôler les mouvements du corps?

Longtemps prédominante, une première hypothèse propose que le cerveau utilise le modèle interne de la gravité pour anticiper et compenser ses effets $[4,5]$. Cette hypothèse, appelée «compensation », présente l'avantage de faciliter le contrôle moteur en s'affranchissant de cette force perturbatrice qui pourrait affecter les trajectoires de nos effecteurs. Un patron moteur unique, par exemple une trajectoire invariante, pourrait être utilisé pour déplacer notre corps dans des situations d'amplitudes, de directions ou encore de durées variées. Une autre hypothèse, nouvelle, propose que notre cerveau utilise le modèle interne de la gravité, non pas pour en supprimer les effets mécaniques sur notre corps, mais, au contraire, pour les utiliser lorsqu'ils lui sont avantageux, afin de minimiser les efforts musculaires.
Cette hypothèse, appelée « optimisation des efforts », repose sur l'observation de trajectoires qui évoluent avec la direction du mouvement par rapport à la verticale gravitaire, et selon l'articulation sollicitée [6]. Ces résultats semblent donc violer l'invariance des trajectoires, proposée par l'hypothèse de compensation.

\section{L'étude des mouvements mono-}

articulaires du bras

Dans de récents travaux, nous avons confronté ces deux hypothèses aux données d'expérimentations permettant de tester spécifiquement le rôle du modèle interne de la gravité dans la production du mouvement [7]. Précisément, nous avons étudié l'organisation temporelle des mouvements mono-articulaires du bras, réalisés dans 17 directions différentes (Figure IA). Les mouvements mono-articulaires permettent d'évaluer les effets spécifiques de la gravité, puisque seuls ces effets changent selon la direction du mouvement: l'accélération gravitaire s'oppose à l'accélération, et s'ajoute à la décélération, d'un mouvement du bras dirigé vers le haut et inversement pour un mouvement vers le bas. Afin de quantifier l'organisation temporelle des trajectoires, nous avons enregistré le déplacement du bras à l'aide d'un système vidéo infra-rouge à haute fréquence $(200 \mathrm{~Hz})$. Nous avons ainsi pu calculer un paramètre pertinent, le ratio de symétrie du profil de vitesse (noté RdS dans la Figure IB), permettant de quantifier l'importance relative des phases d'accélération et de décélération du bras sur la durée totale du mouvement. 
A

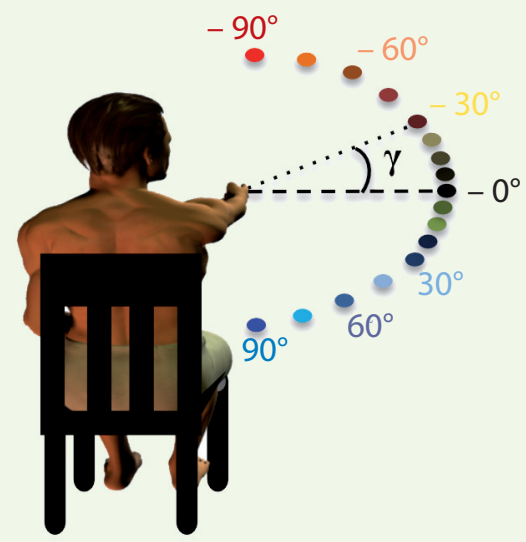

C

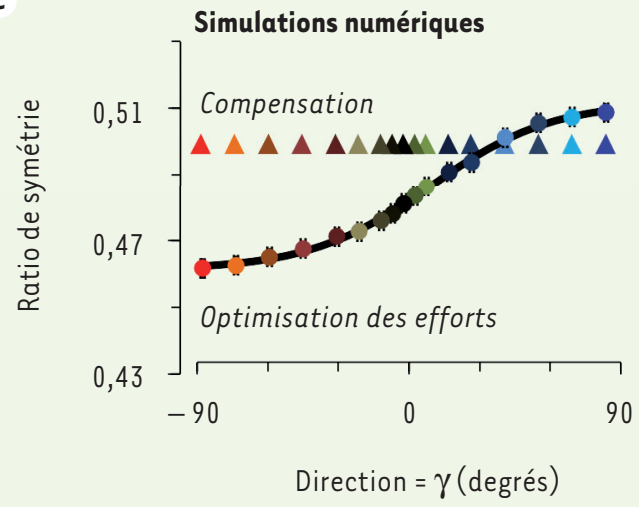

B Compensation
des efforts

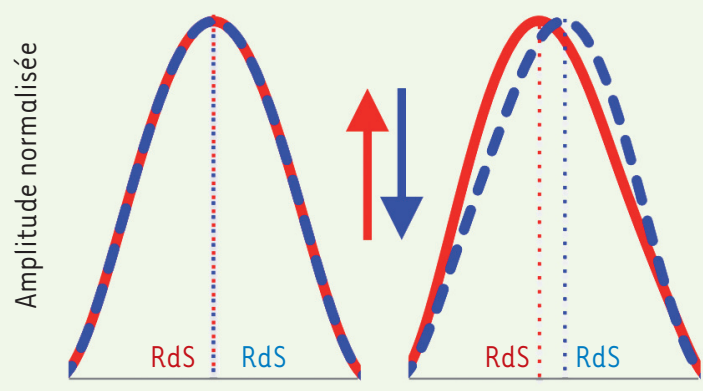

Durée normalisée

D

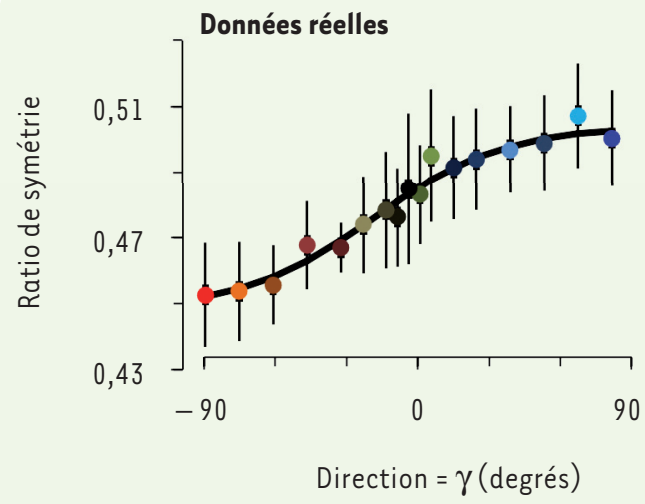

Figure 1. Simulations théoriques des hypothèses et des preuves empiriques. A. Position des participants et projections des dix-sept cibles testées dans le plan frontal. B. Profils de vitesse moyens, normalisés en amplitude et en durée, illustrant les prédictions de l'hypothèse de compensation et de celle d'optimisation des efforts. Le paramètre calculé pour quantifier l'organisation temporelle des trajectoires est le ratio de symétrie du profil de vitesse (RdS: durée d'accélération/durée du mouvement). Les flèches indiquent les deux directions considérées dans cette illustration. C. Ratios de symétrie prédits par les deux hypothèses pour les dix-sept directions de mouvement. $D$. Ratios de symétrie moyens ( \pm écart type) enregistrés chez quinze volontaires sains.

Comme illustré par les simulations numériques présentées dans les Figures $I B$ et $I C$, les hypothèses de compensation et d'optimisation des efforts prédisent des comportements très différents. L'hypothèse de compensation prévoit une trajectoire invariante alors que celle d'optimisation des efforts prédit le suivi d'une trajectoire qui varie de manière sigmoïdale à travers les dix-sept directions. Les données expérimentales recueillies chez quinze volontaires sains révèlent une modulation sigmoïdale de l'organisation temporelle de la trajectoire (Figure ID). Ce résultat s'oppose donc à l'hypothèse de compensation et tend à valider celle d'optimisation des efforts.
En collaboration avec le Centre national d'études spatiales (CNES), afin de tester plus spécifiquement cette hypothèse et pour écarter la possible participation de facteurs périphériques (comme le type de motoneurones et le mode de contraction musculaire), nous avons réalisé une seconde expérience à bord d'un avion effectuant un vol parabolique qui permet de mesurer les effets d'un environnement micro-gravitaire sur la production du mouvement. Si l'hypothèse d'un contrôle moteur minimisant nos efforts dans le champ gravitaire est valide, les différences directionnelles observées sur le ratio de symétrie devraient s'estomper au cours de l'adaptation au milieu micro-gravitaire. La Figure 2 présente les résultats obtenus avec onze volontaires sains ayant réalisé des mouvements de pointage en microgravité au cours de cinq paraboles et dans deux directions (Figure 2A). Conformément à la prédiction de l'hypothèse d'optimisation des efforts (Figure 2B), l'asymétrie de l'organisation temporelle des trajectoires (haut versus bas, en $1 \mathrm{~g}$ ) a progressivement disparu au cours de l'adaptation à la microgravité (Figure 2C; 0 g de P[parabole] 1 à P5).

\section{Intérêt pour les sciences}

et la médecine

Nos résultats indiquent que le cerveau humain a appris à produire des 
A

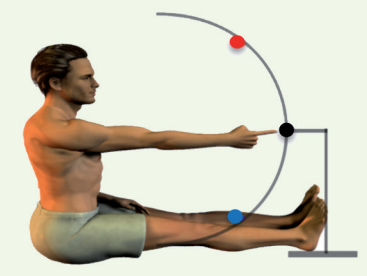

B Simulations numériques

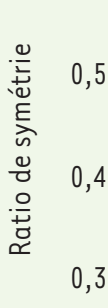

0,55
0,45
0,35
C

Données réelles

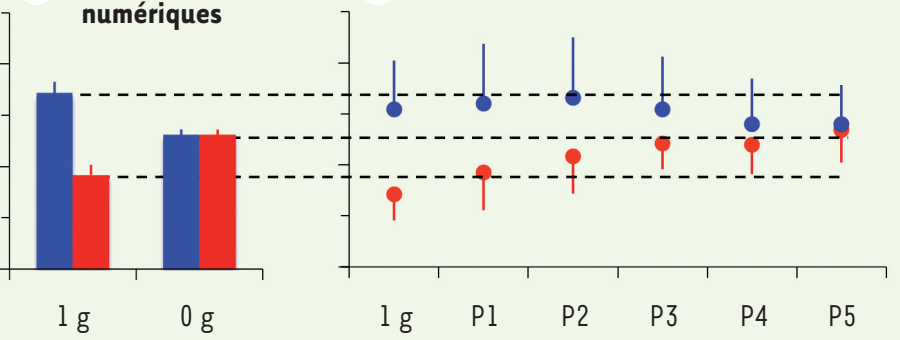

Figure 2. Adaptation à la microgravité. A. Position des participants et position des cibles. B. Ratios de symétrie prédits par l'hypothèse d'optimisation des efforts en situation de gravité terrestre normale $(\mathrm{l} \mathrm{g})$ et en microgravité $(0 \mathrm{~g})$. C. Ratios de symétrie moyens ( \pm écart type) enregistrés chez onze volontaires sains en situation de gravité terrestre normale ( $\mathrm{lg}$ ) et en microgravité au cours de cinq paraboles ( $0 \mathrm{~g}$ de $\mathrm{Pl}$ à P5).

mouvements finement ajustés, exploitant au mieux les effets de la gravité terrestre afin d'économiser nos efforts musculaires. Notre étude réfute l'hypothèse de compensation, encore très influente actuellement [5], et fait progresser notre connaissance fondamentale des mécanismes de production du mouvement par le système nerveux central. Ces mécanismes sont encore mal compris, comme l'illustrent les grandes difficultés auxquelles nous sommes confrontés dans l'élaboration de technologies d'assistance au mouvement ou de robots humanoïdes [8] $(\rightarrow)$.

$(\rightarrow)$ Voir la Synthèse de S. Van Sint Jan Décrypter et antici- et al., $\mathbf{m} / \mathbf{s} \mathbf{n}^{\circ} \mathbf{5}$, per ce que «veut» mai 2013, page 529 faire le système moteur humain est un élément crucial pour ces applications.

Pour la rééducation post-accident vasculaire cérébral, par exemple, de nouveaux protocoles/technologies (un support complet ou partiel du poids des effecteurs) ont été développés à partir de l'hypothèse selon laquelle notre système moteur compense les effets de la gravité [9]. De récents travaux de neurophysiologie suggèrent cependant qu'un support partiel du poids de nos membres altère l'expression des synergies musculaires produisant leurs mouvements [10].

À une époque où le développement technologique est en pleine expansion, il est nécessaire de s'assurer que les nouvelles connaissances/technologies sont orientées et paramétrées de manière à favoriser une interaction avec le système moteur humain qui soit la plus bénéfique possible. Sans une compréhension profonde du comportement moteur, un tel objectif semble inatteignable [11]. $\diamond$ Optimal adaptation of human movement to the gravity field

\section{LIENS D'INTÉRÊT}

Les auteurs déclarent n'avoir aucun lien d'intérêt concernant les données publiées dans cet article.

\section{RÉFÉRENCES}

1. Franklin DW, Wolpert DM. Computational mechanisms of sensorimotor control. Neuron 2011 ; $72: 425-42$.
2. Angelaki DE, Shaikh AG, Green AM, Dickman JD. Neurons compute internal models of the physical laws of motion. Nature $2004 ; 430$ : 560-4.

3. Indovina I, Maffei V, Bosco G, et al. Representation of visual gravitational motion in the human vestibular cortex. Science $2005 ; 308: 416-9$

4. Hollerbach MJ, Flash T. Dynamic interactions between limb segments during planar arm movement. Biol Cybern 1982; 44 : 67-77.

5. Kadmon Harpaz N, Flash T, Dinstein I. Scaleinvariant movement encoding in the human motor system. Neuron $2014 ; 81: 452-62$.

6. Gaveau J, Berret B, Demougeot L, et al. Energyrelated optimal control accounts for gravitational load: comparing shoulder, elbow, and wrist rotations. J Neurophysiol 2014 ; 111:4-16.

7. Gaveau J, Berret B, Angelaki DE, Papaxanthis C. Direction-dependent arm kinematics reveal optimal integration of gravity cues. Elife 2016 ; 5.

8. Van Sint Jan S, Wermenbol V, Van Bogaert P. Une plate-forme technologique liée à la paralysie cérébrale. Le projet ICT4Rehab. Med Sci (Paris) $2013 ; 29$ : 529-36.

9. Prange GB, Jannink MJ, Stienen AH, et al. Influence of gravity compensation on muscle activation patterns during different temporal phases of arm movements of stroke patients. Neurorehabil Neural Repair 2009 ; $23: 478-85$.

10. Runnalls KD, Anson G, Byblow WD. Partial weight support of the arm affects corticomotor selectivity of biceps brachii.J Neuroeng Rehabil 2015 ; 12 : 94.

11. Krakauer JW, Ghazanfar AA, Gomez-Marin A, et al. Neuroscience needs behavior: correcting a reductionist bias. Neuron $2017 ; 93: 480-90$.

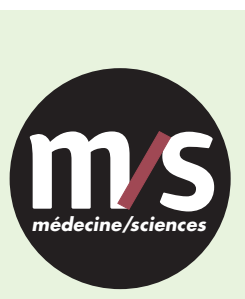

Tarifs d'abonnement $\mathrm{m} / \mathrm{s}$ - 2017

$>$ Grâce à $m / s$, vivez en direct les progrès

des sciences biologiques et médicales

Abonnez-vous

à médecine/sciences

Bulletin d'abonnement page 806 dans ce numéro de $\mathrm{m} / \mathrm{s}$

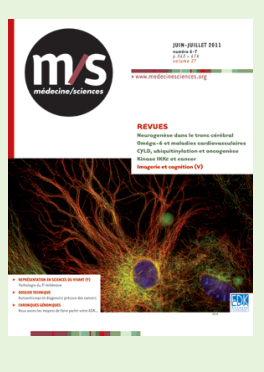

